\title{
Study on the Application of Chinese Traditional Graphics in the Design of Chinatown Guide System
}

Yushan Su

Birmingham City University

Abstract: The overseas Chinatown is the main social and consumption place of local Chinese, the tourist attraction of domestic tourists, and also popular among the local foreigners. Chinatown has the remarkable characteristics of cultural complexity and functional diversity. As a medium of environmental information and human communication, the guide system is particularly significant under the complex urban environment of Chinatown. This paper takes Chinese traditional graphics as the starting point to explore the combination of modern design and Chinese traditional concepts in the guide system, aiming to build a modern urban oriented guide system combined of Chinese cultural characteristics and western culture.

Keywords: Chinese Traditional Graphics; Urban Guide System Design; Chinatown

\section{The application overview of Chinese traditional graphics in the design of Chinatown guide system}

\subsection{Chinese traditional graphics}

Chinese traditional graphics is meant to be after long history accumulation, the pattern and decoration of typical culture with abundant connotation gradually formed by Chinese culture and art, including graphics of figure, plant, animal and totem. It is not only the symbol and expression of the long history of the Chinese nation, but also the inexhaustible source of modern art.

\subsection{The meaning and design of guide system}

There is no unified definition of guide system. Combined with literature review, to sum it up, urban guide system refers to a visual image indication system in the form of words, graphics, symbols, which can clearly express the content, position, direction, safety, rules and other urban functions. Its design based on the way of space cognition of human beings, can help people to recognize, understand and use the systematic design of space through the design of graphics, color, font, composition, material, technology, shape, layout and other factors in the whole urban space.

\subsection{Chinatown guide system}

Chinatown's function has changed a lot from a simple residential area turned into a prosperous tourist area and commercial center. As for the foreigners, Chinatown is an epitome, the first step for them to understand and the guardian of Chinese art, history and tradition. As an essential part of the urban environment layout, all the design elements of the guide system should reflect the urban context, which is the explicit and implicit urban essence connotation precipitated in the process of urban development and the thinking path of the design by analyzing, refining and expressing the context according to the law of visual communication. This paper points out that in addition to giving full play to its basic indicative function, the design of Chinatown's guide system needs to highlight the urban context of Chinatown, which is the characteristics of Chinese culture, by spreading Chinese culture and spirit to improve the overall image of Chinatown.

Copyright $(2020$ Yushan $\mathrm{Su}$

doi: 10.18686/ahe.v4i10.2914

This is an open-access article distributed under the terms of the Creative Commons Attribution Non-Commercial License (http://creativecommons. org/licenses/by-nc/4.0/), which permits unrestricted non-commercial use, distribution, and reproduction in any medium, provided the original work is properly cited. 


\section{The visual application of Chinese traditional graphics in the design of Chinatown guide system}

Guide system design ought to be a systematic interactive design integrating brand image, architectural landscape, traffic nodes and information function sustainability. It can use a variety of visual elements and methods to convey spatial information, forming a three-dimensional, unified urban culture and visual image. The visual elements of guide system design include four aspects: figure, font, layout and color, which should be the basic rule when designing various cultural characteristics of traditional Chinese figures, so as to form a city guide system equipped with Chinese cultural characteristics, easy to spread, recognize and remember, free of communication and meet the complex urban environment of Chinatown.

\subsection{Graphic-Show the appearance, get the meaning and share the spirit}

The graphic design of the guide system is the key to reflect the function of easy to identify and remember. It can convey the information to the public more quickly and accurately. Comparatively, it is more intuitive than the text, with more abundant information. Therefore, it is the basic rule of urban guide system design to carry out innovative design of guide system's graphic symbols to achieve the unity of functionality, culture and artistry. We need to find more graphic elements to highlight the role of guide. Chinese traditional pattern has a long history, which is not only a decorative pattern, but also a pattern of Chinese spirit. It contains deeper symbolic meaning and philosophical connotation, and has great emotional communication. For an instance, some of the most representative patterns of Chinese traditional patterns are The Panchang Figures: Referring to the continuous implementation of the loopback, extends the good wishes for the prosperity of the family, the continuation of the offspring, the wealth and auspiciousness of the generations. The Chinese knot, we are familiar with, is the evolution of the Panchang Figures and the most famous application is the symbol of China Unicom. Like the dragon, Phoenix has become the most vital cultural symbol and spiritual symbol of the Chinese nation after thousands of years of historical and cultural baptism and the most famous application is the logo of Phoenix Television.

Compared with the western scientific and regular design style, by the way of showing the appearance, getting the meaning and sharing the spirit, Chinatown's guide system design can extract and modernize the representative elements of Chinese traditional patterns, and then apply to the design to achieve emotional communication and exchange as well as the embodiment of the depth of Chinese culture, meanwhile, to achieve efficient cognition and integrate into the space.

\subsection{Font-Chinese characters in graphical format}

Character is the basic element of guide design. The design of font can give guide system more artistic and cultural implications. As the most representative cultural symbol in China, there is no doubt that Chinese characters are widely used in Chinatown's guide system. Chinese characters in graphical format is one of the main forms of Chinese character design, based on the structure, stroke and meaning of Chinese characters, in a creative process of visualization and symbolization by means of meter, rhythm, direction, virtual reality and other techniques of formal beauty. In terms of the complexity of the citizens in Chinatown, it is particularly important to use the graphic Chinese characters in the guide system.

As far as I am considered, the graphic design of Chinese characters can follow the original method of design. The socalled originality refers to the origin of modern Chinese characters which originated and evolved from original pictures. They are close to pictures but not exact pictures and close to words but not exact words.

The original method of design can be combined with the ancient hieroglyph, the meaning of the word itself or the concrete graphic symbols, so as to realize the purpose of understanding the text freely. At the same time, in the design of Chinatown's guide system, we can also use the traditional Chinese calligraphy and seal cutting techniques of Chinese characters. Calligraphy, one of the most refined and respected forms of Chinese art, has a strong sense of figure with several simple black lines to express the vivid brushwork and charm. Seal cutting is more outstanding in the design and application of Chinese characters, combining calligraphy and sculpture. Among a lot of contemporary designs, seal cutting is one of the most commonly used design elements that embody Chinese special characteristics.

\subsection{Layout-Combination of grid and traditional composition}

One of the key design elements in the guide system is layout design whose quality determines whether the audience can better read the effective content and the fluency of reading efficiency. It must cater to the audience's recognition habit and aesthetic taste. In other words, with clearer level, more reasonable collocation and more abundant artistic beauty, the function of the guide system can be maximized.

In the modern guide system design, the grid is widely used. The designers fill in the grid with rich design elements, to 
bring a sense of proportion and order, so as to achieve the coherence and overall unity of the guide reading habit. Throughout the composition layout of traditional Chinese patterns, its Two sides continual, Four sides continual, S-shaped composition, grid composition, and the“阊”composition, are similar to but thousands of years earlier than the grid design, which originated in Western Europe in the early 20th century and improved in Switzerland in the 1950s. The layout design of the guide system of the Chinatown can source inexhaustible design elements from the composition layout of traditional Chinese patterns. For example, the bronze patterns from the Spring and Autumn Period are mainly Two sides continual and Four sides continual. In terms of composition, they are arranged in the form of bilateral symmetry. The open space of patterns is covered with many square continuous thunder patterns, resulting in a sense of visual stability and decoration.

In the design of Chinatown's guide system, we can combine the composition form of Chinese traditional pattern with the grid design appropriately and design a series of continuous unified graphics containing the guide function in the global vision, which is not a simple graphic repetition, but a design of different meanings through the transformation of color and graphic laws.

\subsection{Color-Innovative application of five color system}

The color application scheme is an important part of the guide system design, which has great visual rendering, decoration and beautification functions and attractive information communication ability. Meanwhile, different colors can show brand positioning and cultural implication, leaving a deep impression on the audience. According to the different characteristics of the environment and the design object, the visual effect and the purpose of the guide system, the design should reasonably match and combine colors and follow three color design principles: functionality, aesthetics and culture.

As the embodiment and inheritor of Chinese culture, the color application of Chinatown's guide system should be able to highlight Chinese characteristics and reflect the spirit and connotation of China. The five color system of Chinese traditional colors: red, yellow, green, black and white, has strong national cultural consciousness and humanistic art concept. We can establish 2 or 3 kinds of them as the main colors of Chinatown's guide system, and in the meantime, use the color collocation and expression techniques in Chinese traditional patterns for reference to realize the innovative design of color application scheme. When determining the main color, firstly, we ought to understand the unique symbolic meaning of each color. Red is the symbolic color of the Chinese nation and the symbol of the sun, which represents the meaning of warmth, prosperity, auspiciousness and so on. Black, is the most primitive color perception inherited by China. And the other one represents purity, brightness and fullness. The color matching method of "two color tone" in traditional Chinese patterns is an important feature, which has representative black and white in Chinese paintings, blue and white in blue and white porcelain, red and black in porcelain of Han Dynasty, and black and white in Taiji with the most traditional Chinese philosophy significance. This two-color matching seems simple, but it expresses clear artistic conception, and can create thousands of changes, but it is harmonious and unified with visual effect of rules to be followed, giving people a distinct impression. In Chinatown's guide system, we can analyze the relationship between color, text, figure and context. Based on the color matching method of "two color tone", combined with the modern design concept, we can form a color application scheme that is distinct and easy to identify, which is consistent with Chinatown's urban context.

\section{References}

1. Zhang R. The inheritance and development of Chinese traditional patterns in modern logo design. M.Sc. Thesis. Tsinghua University; 2004.

2. Ren L. Traditional Chinese visual design elements: Their applicability in contemporary Chinese design. A thesis presented in partial fulfillment of the requirements for the degree master of science in design. Arizona State University; 2013.

3. Sun L. Study on the application of Chinese traditional patterns in modern graphic design. International Conference on Arts, Design and Contemporary Education; 2016.

4. Li J. Exploration and innovation of traditional graphic symbols. M.Sc. Thesis. Wuhan University of Technology; 2008.

5. Hou JJ. The application of traditional graphics in the guide system design-Take the guide system design of tai'an culture square as an example. M.Sc. Thesis. Beijing Institute of Graphic Communication; 2017.

6. Calori C. Signage and way finding design: A complete guide to creating environmental graphic design systems hoboken, N.J.: John Wiley; 2007.

7. Mollerup P. Way showing > Way finding: basic and interactive Amsterdam: BIS Publishers; 2013. 